\title{
3 Research Square

\section{Trophic states regulate assembly processes and network structures of small chromophytic phytoplankton communities in subtropical coastal ecosystem}

\section{Xing Chen}

Nanning Normal University https://orcid.org/0000-0002-7270-7051

Huaxian Zhao

Nanning normal university

Gonglingxia Jiang

Nanning normal university

Jinli Tang

Nanning normal university

Qiangsheng Xu

Nanning normal university

Si Chen

Guangxi university

Shuqi Zou

Kyonggi university

Ke Dong

Kyonggi University

\section{Weiguo Hou}

China University of Geosciences

\section{Lianghao Pan}

Guangxi Mangrove Research Center

\section{Pengbin Wang}

Key Laboratory of Marine Ecosystem Dynamics, Second Institute of Oceanography, Ministry of Natural Resources

Nan Li ( $\square$ nli0417@163.com )

Nanning normal university https://orcid.org/0000-0003-1782-6610

\section{Research Article}


Keywords: Small chromophytic phytoplankton (SCP), rbcL, Co-occurrence network, Assembly process, Eutrophication

Posted Date: October 29th, 2020

DOI: https://doi.org/10.21203/rs.3.rs-55561/v3

License: (c) (1) This work is licensed under a Creative Commons Attribution 4.0 International License. Read Full License 


\section{Abstract}

Small chromophytic phytoplankton (SCP) are anticipated to be more important for a significant proportion of primary production in estuarine-coastal ecosystems. However, responses of SCP community to coastal eutrophication are still unclear. In this study, we investigated diversity, cooccurrence and assembly features of SCP communities, as well as their relationship with environmental factors in the subtropical Beibu Gulf. The results exhibited that the alpha diversity (ANOVA, $p<0.001$ ) and beta diversity (ANOSIM, $p<0.001$ ) of SCP communities were significantly different among eutrophic states. Co-occurrence network analysis revealed a complex interaction between SCP community and environmental factors. Most ASVs in modules of the network were specific to trophic states. Further, phylogenetic based $\beta$-nearest taxon distance analyses revealed that stochastic processes mainly provided $69.26 \%$ contribution to SCP community assembly, whereas deterministic processes dominated community assembly in a heavy eutrophic state. Importantly, increased environmental disturbances, such as nitrogen and phosphorus nutrients, could alter SCP community structure and disrupt ecological processes. Overall, our findings elucidate the mechanism of diversity and assembly in marine SCP community and promote the understanding of SCP ecology related to subtropical coastal eutrophication.

\section{Introduction}

Anthropogenic activities and climate changes impose severe effects on aquatic coastal ecosystems with the advent and expansion of coastal aquaculture, urban, agricultural, and industrial runoff (Escalas et al., 2019). As an important component in aquatic coastal ecosystems, marine small chromophytic phytoplankton (SCP) that defined as cells within the size range of $0.2-20 \mu \mathrm{m}$, including both picophytoplankton and nanophytoplankton, have an advantage over larger cells in nutrient uptake due to their higher surface to volume ratio (Irion et al., 2020; Irwin et al., 2006). They are vital contributors of primary production for its photosynthetic component and perform a range of ecological roles in carbon fixation, nutrient cycling and regulating overall food-web dynamics (Bolaños et al., 2020; Capblancq, 1990; Falkowski et al., 1998). Furthermore, SCP generally become an important indicator of ecological change in marine ecosystems responding to environmental perturbation (Almanza et al., 2019; Beaver et al., 2018; Monchamp et al., 2018).

Modern molecular methods have revolutionized our knowledge of assessment of marine SCP communities and diversity. For example, 18S rRNA (for pico-eukaryotes) (Irion et al.; Staay et al., 2000) represent useful tools for ecologists to study diversity. Besides, functional gene markers, especially $r b c L$, which encodes the large subunit ( $L$ ) of ribulose-1,5-bisphosphate carboxylase/oxygenase (RubisCO) involved carbon assimilation (Nan et al., 2016; Xu and Tabita, 1996), have been extensively used and provide increased resolution for assessments of biodiversity on SCP community across different coastal environments, including the bay of Bengal (Pujari et al., 2019), Western Iberia (Ferreira et al., 2019), Western Mediterranean (Paches et al., 2019) and Northern South China Sea (Li et al., 2016). Although the general patterns underlying variations in biodiversity have been observed across spatial and temporal 
scales, the response mechanism of SCP to environmental changes in eutrophic seawater are still large unknow.

Most importantly, increasing evidences suggest that intrinsic mechanisms and interactions markedly play critical roles in phytoplankton community's response to variations in external environmental factors (Wentzky et al., 2020; Xue et al., 2018; Yan et al., 2020). For example, total nitrogen (TN) determined species co-occurrences pattern, making a substantial phytoplankton compositional shift towards diatoms in the reservoir (Ribeiro et al., 2018). In eutrophic condition, phytoplankton communities under cyanobacteria dominance exhibited a lower cohesion and less interactions between taxa but at nonetheless compositionally stable (Escalas et al., 2019). So far, the impacts of different eutrophic states on the intrinsic interactions and structure of SCP networks remain unclear.

Moreover, environmental factors can also intensively influence the assembly mechanisms (deterministic and stochastic processes) that are involved in jointly shaping phytoplankton community variation (Chen et al., 2019; Gao et al., 2020; Hanson et al., 2012). A previous study proposed that total phosphorus (TP) was significantly correlated to the microeukaryotic communities, which may shape microeukaryotic community assembly in a subtropical river across wet and dry seasons (Chen et al., 2019). The other studies suggested that nutrient input contributed to a gradual shift from stochastic to deterministic-based processes of microbial communities in fluidic ecosystems (Van Der Gast et al., 2008; Zhou et al., 2014). However, there exists limited knowledge on assembly processes of SCP community in marine eutrophic environment.

Here, we explored the diversity of SCP communities using the $r b c L$ gene based on high-throughput sequencing in the subtropical gulf, which was strongly affected by anthropogenic activities (Ding et al., 2019; Xu et al., 2019). The goals of this study were to (i) determine how the diversity and composition of the SCP community vary along eutrophic gradients in coastal ecosystems, (ii) uncover the co-occurrence patterns of SCP communities, and (iii) explore the underlying nutrient factors regulating community assembly processes along eutrophic gradients.

\section{Materials And Methods}

\subsection{Sampling Sites and Environmental Parameters}

A total of 90 water samples were collected across three predefined sampling areas encompassing Maowei Sea estuary (H1-H4 sites), adjoining Qinzhou Bay (M1-M6 sites) and Beibu Gulf open sea (01-08 sites), with 5 duplicates per site. All samples were collected within two weeks to negate the effects of seasonality on SCP community during October, 2018. Each sample was collected at a depth $5 \mathrm{~m}$ using a sterilized water-sampling device and immediately transferred to the laboratory within $24 \mathrm{~h}$ for further processing. The $10 \mathrm{~L}$ seawater of each sample was prefiltered using 100- $\mu \mathrm{m}$ nylon mesh (Millipore, U.S.A.) in order to remove large particles. Then filtering successively through 20 and $0.2-\mu \mathrm{m}$ pore-size membranes (Millipore) for small size fractions using a vacuum pump. Then, the filters were immediately 
stored in sterile $50 \mathrm{~mL}$ centrifuge tubes at $-20^{\circ} \mathrm{C}$ until DNA extraction. At the time of sampling in each station, a portable meter (556 MPS; YSI, USA) was used to measure seawater salinity, pH and temperature. Additionally, other environmental parameters including dissolved inorganic nutrients $\left(\mathrm{NO}_{2}{ }^{-}\right.$, $\mathrm{NO}_{3}{ }^{-}, \mathrm{NH}_{4}{ }^{+}$, and $\mathrm{PO}_{4}{ }^{3-}$ ) measured by spectrophotometric and colorimetric methods (Han et al., 2012), the total organic carbon (TOC) and chemical oxygen demand (COD) measured by the standard method (Visco et al., 2005), the total dissolved nitrogen (TDN) and total dissolved phosphorus (TDP) determined by the Cu-Cd column reduction and spectrophotometric phosphomolybdate blue method, respectively (Cai and Guo, 2009), and Chl a measured by a fluorescence spectrophotometer (Holm-Hansen and Riemann, 1978) were recorded. Dissolved inorganic nitrogen (DIN) and dissolved inorganic phosphorus (DIP) were composed of $\mathrm{NO}_{2}{ }^{-}, \mathrm{NO}_{3}{ }^{-}, \mathrm{NH}_{4}{ }^{+}$and $\mathrm{PO}_{4}{ }^{3-}$, respectively. The eutrophication index (EI) was calculated as: EI=DIN×DIP×COD×105/4500 (Dai et al., 2017). Eutrophic states were categorized as follows: oligotrophication ranged from $1.0<\mathrm{El}<3.0$; medium eutrophication ranged from $3.0<\mathrm{El}<9.0$; and heavy eutrophication ranged from El > 9.0 (Dai et al., 2017). We determined the that trait distribution of eutrophication showed a decreasing trend from the Maowei Sea to Beibu Gulf open sea. Consequently, sampling sites were divided into three groups: heavy eutrophication (HE), medium eutrophication (ME) and oligotrophication (OE).

\subsection{DNA Extraction, PCR Amplification and Sequencing}

Environmental DNA extraction was performed using a DNeasy Power Water Kit (TIANGEN, China) through $0.2-\mu \mathrm{m}$ pore size polycarbonate membranes according to the manufacturer's protocols. DNA yield and purity were evaluated using a Nanodrop-2000 Spectrophotometer (Thermo Scientific, USA). The DNA sample was preserved at $-80^{\circ} \mathrm{C}$. Form ID $r b c L$ gene fragment (550 bp) was amplified from environmental DNA for all the stations using previously published rbcL primers (F:5'-GATGATGARAAYATTAACTC-3' and R:5'-ATTTGDCCACAGTGDATACCA-3') (John et al., 2007). PCR reactions were conducted with $2 \mu L$ DNA

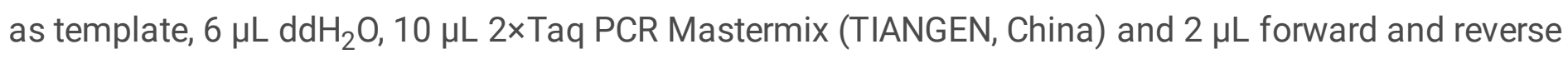
primers per $20 \mu \mathrm{l}$ PCR reaction. PCR cycling conditions were set for $95^{\circ} \mathrm{C}$ for $1 \mathrm{~min}$, followed by 35 cycles of denaturation at $95^{\circ} \mathrm{C}$ for $30 \mathrm{~s}$, annealing at $56^{\circ} \mathrm{C}$ for $30 \mathrm{~s}$, extension at $72{ }^{\circ} \mathrm{C}$ for $30 \mathrm{~s}$, with a final extension of $72^{\circ} \mathrm{C}$ for $10 \mathrm{~min}$. The PCR products were run on an electrophoresis gel along with a negative PCR control to verify that the PCR products were not contaminated.

Sequences were performed on the Illumina MiSeq platform of Lianchuan Bio-Technology Company (China). Low quality reads, primers, barcode sequences, and chimeric sequences were eliminated to minimize the effects of random sequencing error by DADA2 denoising method of software QIIME2 (Caporaso et al., 2010). Amplicon sequence variants (ASVs) were obtained from Illumina-scale amplicon data without imposing the arbitrary dissimilarity threshold (Callahan et al., 2017). Taxonomic assignments were performed from an available $r b c L$ sequence database generated from GenBank data. Besides, we filtered out sequences that clustered into large phytoplankton, such as Bangiaceae, Pyropia, 
and Ditylum. RbcL gene sequences data were deposited in the NCBI BioProject database under the PRJNA645508.

\subsection{Statistical analysis}

Group differences among the various environmental variables and a-diversity index were tested with a permutation test (number of permutations $=10,000$ ) using the One-way analysis of variance (ANOVA) in SPSS Statistics v24.0 (Park, 2009). The a-diversity of each sample between different groups was determined by calculating Shannon, Simpson, Chao1 and Observed numbers index and calculated using 'vegan' R package (Dixon, 2003). Spearman's rank correlation between the a-diversity index and environmental factors was calculated using 'psych' R package (Revelle and Revelle, 2015) with $P$-value adjusted by the FDR correction. The community composition was analyzed to investigate the variation of relative abundance and visualized using the 'pheatmap' $R$ package (Kolde, 2012). To investigate successional patterns of SCP communities between groups, the $\beta$-diversity was undertaken using BrayCurtis dissimilarity index and visualized by Nonmetric multidimensional scaling (NMDS). The analysis of similarities (ANOSIM) was performed to analysis similarities variation in community using the 'vegan' $R$ package. Correlations between SCP community and constraining variables were tested using Mantel tests between Bray-Curtis distance matrices of community composition and Euclidean distance matrices of environmental factors. Linear regressions were performed to determine the relationships between the Bray-Curtis similarity of SCP community and geographical distance, environmental factors, respectively.

\subsection{Co-occurrence network analysis}

Only ASVs in at least five samples with more than 20 sequences in SCP community were selected to construct co-occurrence networks, as previous study (Hou et al., 2020). To identify correlation between ASVs with trait distributions, spearman's rank correlations were calculated as being positively or negatively correlated with a certain trait using "Hmisc" R package (Harrell Jr and Dupont, 2006). Only robust $(|r|>0.6)$ and statistically significant (FDR-adjusted $P$-value $<0.05)$ correlations were selected for network analysis. A GML format network file was generated using the "igraph" R package and visualized using Gephi 0.9.2 (Csardi and Nepusz, 2006; Johnson, 2019). Networks are composed of nodes, which correspond to individual taxon, connected by links (or edges), that represent significant co-occurrence relationships. Nodes with high degree $(>65)$ are recognized as potential keystone species of the state in co-occurrence networks. The network-level (average node degree, clustering coefficient, average path length, and modularity) topological features of a network was calculated. Meanwhile, the real networks were compared with 1000 Erdös-Réyni random networks, which had the identical number of nodes and edges as the real networks, were generated in the "igraph" R package (Bollobas, 1984). We also tested whether environmental factors related to its modularization in the network using correlation tests in $\mathrm{R}$.

\subsection{Analysis of SCP community assembly processes}


To assess the dominance of stochastic and deterministic assembly processes along the eutrophic gradients, we compared all possible pairwise comparisons of $\beta$-nearest taxon index ( $\beta N T I)$ within certain group and chemical properties ranges with 999 randomizations in the "picante" R package. If $\beta N T I$ values are $>2$ or $<-2$, the dominance of deterministic processes in shaping the community composition, whereas $\beta N T I$ values are between -2 and 2 , community assembly was dominated by stochastic processes. To further evaluate the variation in community assembly processes along nutrient gradients, nearest-taxon index (NTI) and $\beta N T I$ values were regressed against Euclidean distance matrices of nutrition factors. We assessed statistical significance of the resulting comparison by Mantel tests with 999 random permutations to determine the relationships between the $\beta \mathrm{NTI}$ and the Euclidean distance of environmental factors.

\section{Results}

\subsection{Nutrient levels at locations}

We measured a number of climatic and nutrient factors to reveal the extent of environmental heterogeneity across sampling sites (Table S1). The environmental parameters of the seawater samples all exhibited characteristic differences and varied significantly between the stations sampled from various groups (one way-ANOVA analysis, $p<0.001$ ) (Table S2). The DIN was comparable between the Maowei Sea $(0.564 \pm 0.091 \mathrm{mg} / \mathrm{L})$ and Qinzhou Bay $(0.160 \pm 0.057 \mathrm{mg} / \mathrm{L})$. In addition, the El dropped sharply (ranged from 0.55 to 25.48 ) and tended to decrease from the estuary to the open sea area. The lowest value was noted at the Beibu Gulf open sea (1.189 \pm 0.322$)$, and the highest value was observed at the Maowei Sea $(16.380 \pm 5.152)$ (Table S1 \& Figure 1b), which is consistent with the change in DIP. These results suggested that nutrient levels were markedly different among the locations that were divided into three groups that were based on similar intensities of anthropogenic activity, such as intensive aquaculture. Moreover, other environmental parameters at the time of sampling for all the stations are detailed in Table S1.

\subsection{Alpha diversity of the SCP community across eutrophic states}

Across all water samples, we obtained 6,299,765 high-quality sequences after normalizing that were clustered into a total of 855 ASVs after quality control (Table S3). Good's coverage ranged from $99.79 \%$ to $100 \%$ in all the samples, indicating that the current sequences reflected the actual situation of the majority SCP community. The richness and diversity of SCP communities in different groups were compared based on ASV richness and phylogenetic diversity. a-diversity, as expressed by the Shannon and Simpson diversity indices, was the highest in the HE and decreased along eutrophic gradients. We also found that a-diversity were significantly altered between groups (ANOVA analysis, $p<0.001$ ), and remarkable differences were observed in these two indices between HE and OE (Figure 2). However, no significant differences were noted within Chao1 and Observed number indices. Furthermore, the effects 
of geographical distance and other environmental factors on the alpha diversity (Shannon and Simpson indices) of the SCP community were compared using contours map and Spearman's correlation, respectively (Figure 1c \& Table S4). According to the Spearman's correlation analysis, both ASV richness and alpha diversity of the SCP community were significantly negatively $(p<0.01)$ correlated with salinity $(r=-0.46), \operatorname{TOC}(r=-0.43), \mathrm{pH}(r=-0.42)$, and COD $(r=-0.39)$ but also positively $(p<0.001)$ correlated with $\mathrm{NH}_{4}{ }^{+}(r=0.58)$, DIP $(r=0.52)$, TDP $(r=0.5)$, and DIN $(r=0.42)$. Furthermore, El was consistently the best predictor for Shannon diversity with a strong positive significant correlation $(r=0.58, p<0.001)$ (Table S4).

\subsection{Composition and beta diversity of the SCP community across eutrophic states}

The dominant phyla included Bacillariophyta, Cyanobacteria, and Dinophyta, accounting for more than $80 \%$ of the total sequences. The group related to heavy eutrophication was significantly over-dominated in the genera of Thalassiosira, Pseudo-nitzschia and Skeletonema, whereas the oligotrophication groups were mostly affiliated with Thalassiosira, Minutocellus, Navicula, and Chaetoceros. Nonmetric multidimensional scaling (NMDS) analysis based on Bray-Curtis dissimilarity showed that the SCP community compositions derived from three groups exhibited a clear separation across the eutrophication gradient, with samples being more dispersed at oligotrophic level (Figure 3a). A higher beta diversity was found in the OE compared with the other two groups. This pattern was further supported by the results of an analysis of similarity (ANOSIM, R $=0.863, p=0.001$ ), which demonstrated that the Bray-Curtis dissimilarity rank of between group was higher compared with that for each of the three subgroups. These results indicated that the difference in SCP community composition was more significant between groups than within groups (Table S5 \& Figure 3b). Moreover, we also calculated the effects of environmental variables on the beta diversity of SCP community using Mantel tests (Table 1). The results suggested that almost all environmental factors were strongly correlated with SCP community structure, and shifts in SCP community structure may be due to main environmental factors, such as DIP $(r=0.520, p<0.001)$, TDP $(r=0.520, p<0.001)$, geographic distance $(r=0.488, p<0.001)$, temperature $(r=0.447, p<0.001)$, and $\mathrm{pH}(r=0.435, p<0.001)$ (Table 1). The results revealed that $P$ nutrient changes maintained stronger effects on the SCP community composition and exhibited a significant positive relationship with differences in Bray-Curtis dissimilarity which indicates that the larger the $\mathrm{P}$ nutrient difference, the more dissimilarity between the SCP community structure. To gain further understanding of the linkages between SCP community dissimilarity and nutrient changes, we explored linear regressions based on Bray-Curtis distance (Figure 4). The results showed that geographic distance is an important factor that elicits variation in SCP community structure which had a strong positive linear relationship with Bray-Curtis dissimilarity (regression, $\mathrm{R}^{2}=0.412, p<0.001$ ) (Figure 4a). DIP and TDP (regression, $\mathrm{R}^{2}=0.594, p<0.001$ ) also had significant effects on SCP community structure, and the effect of phosphorus level was stronger than that of geographic distance (Figure 4). Overall, these observations strongly suggested that nutrient levels, especially phosphorus, were major drivers in shaping 
the structure and diversity of the SCP community. Notably, the dissimilarities among the SCP communities were also significantly correlated with eutrophic states (Figure 4d), suggesting that the magnitude of changes in SCP communities is dependent on the selective pressures exerted by increasing eutrophication.

Given that SCP communities were dramatically altered by increasing eutrophication, we then asked whether SCP assemblages could be indicative of eutrophic states. Thus, we screened top 10 SCP genera for which their relative abundances were significantly associated with El as depicted in the heatmap (Figure 5). However, the responses of these assemblages to increasing eutrophication were divergent. The majority of SCP genera was significantly different as demonstrated nutrient gradient distribution patterns. For example, assemblages affiliated with Chaetoceros, Navicula, Nitzschia, and Minutocellus were significantly negatively correlated with eutrophic states. All assemblages were negatively related to $\mathrm{El}$, showing a decreasing trend from oligotrophication to high eutrophication (Figure 5). These results suggested that the variation in SCP community varied markedly greater across eutrophic levels. Thus, these sensitive assemblages have the potential to be used as bio-indicators of eutrophic states.

\subsection{Co-occurrence patterns of the SCP community and environmental variables}

In addition to simple composition and diversity, co-occurrence network analysis can potentially provide a deep and unique perspective on SCP interactions and ecological assembly rules that are constructed based on Spearman's rank correlation (Figure 6). The SCP co-occurrence networks consisted of 240 nodes linked by 4432 edges (Table S6 \& Figure 6c). For the SCP co-occurrence network, Thalassiosira (22.12\%), Skeletonema (14.16\%), Chaetoceros (13.27\%), Navicula (5.75\%), Guinardia (4.87\%), Pseudonitzschia (3.98\%), Minutocellus (2.21\%) mainly occupied the nodes (Figure 6b). In the SCP co-occurrence network, 30 ASVs with high degrees and eigenvector centrality were identified as keystone species, and these species were significantly preferred given that almost all were distributed mainly in the OE group (Figure S1). Given that ASVs from the same module co-occurred more frequently than nodes in other modules, co-occurrence network analysis also shows that nodes from the same genus may play an important role in determining modular structures. Consequently, the SCP network was clearly divided into four major modules, of which modules I, II, III, and IV accounted for $34.58 \%, 43.33 \%, 16.37 \%$, and $6.67 \%$ of the whole network, respectively (Figure 6a). Module I and III were primarily occupied by Thalassiosira, Chaetoceros, Navicula, and Skeletonema, whereas module II was mainly composed of Thalassiosira, Skeletonema, and Guinardia. Ternary plot analysis suggested that these modules were specific to a particular eutrophic group (Figure S2). For example, modules I and III were specific to the OE group, whereas modules II and IV were specific to the HE group and ME group. Furthermore, the observed modularity, clustering coefficient and average path length were all greater than those of their respective Erdös-Réyni random networks, indicating the SCP network was non-random with "small-world" properties and modular structure (Table S6). For the integrated networks, the variation of correlations was also significantly correlated with the Euclidean distance of environmental factors. Salinity and pH were mainly 
attached to module I, which was associated with oligotrophication, whereas nutrients clustered into module II were associated with heavy eutrophication. DIP, TDP, and El exhibited higher degrees and eigenvector centrality compared to other environmental factors, indicating that nutrient levels had stronger relationships with SCP ASVs compared to other environmental factors in the Beibu Gulf (Figure $6 c)$.

\subsection{Assembly processes of SCP communities along eutrophic gradients}

To discriminate between the deterministic and stochastic processes in shaping SCP community along eutrophic gradients, we compared the different assembly processes of SCP community in all samples and the three groups using within-community (nearest-taxon index [NTI]) and between community (BNTI) assessments (Figure 7). For the overall group, stochastic processes, which encompassed dispersal limitation (10\%), homogenizing dispersal (36.34\%), and ecological drift (22.92\%), contributed a larger fraction $(69.26 \%)$ to the community assembly, indicating that stochastic processes dominated community assembly. In contrast, deterministic assembly processes (80\%) mainly influenced the SCP community in the HE group. In different groups, the relative contribution of stochastic processes varied from $20 \%$ in $\mathrm{HE}$ and $87.59 \%$ in ME to $99.23 \%$ in OE. We also found the NTI values decreased linearly in relation to increasing DIN and El, indicating that the community assembly tended to strengthen significantly with increasing eutrophication (Figure 8a \& 8c). To infer alterations in the deterministic/stochastic assembly processes along nutrients gradients, we examined the relationships between $\beta N T I$ and environmental variables using Mantel test analyses (Table 3). Pairwise comparisons of $\beta N T I$ values for the SCP communities were mainly significantly positively correlated to changes in DIN $(r=0.665, p<0.001), \mathrm{NO}_{3}{ }^{-}(r=0.635, p<0.001)$, salinity $(r=0.520, p<0.001)$, El $(r=0.520, p<0.001)$, $\mathrm{NH}_{4}{ }^{+}(r=0.469, p<0.001)$ and temperature $(r=0.300, p<0.001)$, indicating that increases in their divergence were associated with a shift in SCP community assembly processes from stochasticity to heterogeneous selection (Table 2). Additionally, the deterministic processes that shaped the community assembly in the HE group tended to strengthen significantly (Mantel-High, El, $r=0.966, p<0.001$ ) with increasing coastal eutrophication. Overall, the regression models showed that most of the $\beta N T I$ values were between -2 and 2 , indicating a dominant role of stochasticity in the entire SCP community (Figure $8 \mathrm{~b}$ $\& 8 d)$ that was significantly affected by nitrogen concentration, salinity, and temperature.

\section{Discussion}

\subsection{Characteristics of the SCP communities with increasing eutrophication}

Increasing evidence has shown that disturbance could select species exhibiting specific biological traits and markedly alter the composition of SCP community (Li et al., 2020; Stegen et al., 2012). A high relative 
abundance of Thalassiosira, Pseudo-nitzschia and Skeletonema, were observed and widely spread in the heavy eutrophication Maowei Sea, whereas Thalassiosira, Minutocellus, Navicula, and Chaetoceros were significantly over-dominated in the Qinzhou Bay and Beibu Gulf open sea as eutrophic states decreased, which is consistent with previous studies (Leruste et al., 2019; Tian et al., 2017). Bacillariophyta exhibited high adaptability for oligotrophic conditions as to be dominant in nutrient-deficient areas (Zhou et al., 2017). This assertion is supported by our previous finding that nutrient variables exhibited clear spatially structured patterns. Additionally, the variation in the relative abundance of top 10 SCP genera closely correlated with eutrophic states, indicating that sensitive assemblages could be considered as bioindicators for evaluating eutrophic states (Figure 5). For example, the high relative abundance of oligotrophic assemblages has been extensively detected and was negatively correlated with El (Figure 5). In general, changes in the patterns of these sensitive assemblages were significantly associated with eutrophic states, which is consistent with their ecological strategies. As previous study described (Pujari et al., 2019), certain SCP community may be missing due to the bias of the primers used for PCR amplification. Besides, the discrepancy of region that make certain SCP discovered in other studies (Bolaños et al., 2020; Irion et al.), such as Haptophyceae, Pelagophyceae, and Cyanophyceae, to be inevitably left out in the Beibu Gulf.

\subsection{Drivers of alpha and beta diversity in SCP communities}

In this study, we used Spearman's correlation to investigate the effects of environmental variables on the SCP community and found that Shannon and Simpson diversity exhibited significant positive relationships with nitrogen and phosphorus concentration but negative relationships with $\mathrm{pH}$ and salinity (Table S4). There are two possible explanations for such significant effects. One possible explanation for this positive effect could be attributed to the fact that the accumulation of nutrients increased the nutritional pressure to select tolerance of many species, thereby increasing the community alpha diversity. The high diversity in the HE group may increase the functional redundancy of the SCP community and further provide biological buffering capacity to resist environmental changes (Yachi and Loreau, 1999). Another possible explanation for this negative effect was that the increasing salinity prevented taxa from adapting to the enhanced osmotic pressure, so these taxa may die or become inactive (Oren, 2011), thus reducing alpha diversity.

Consistently, we found that increasing eutrophic states drove gradually stronger changes in picoplankton community composition and exhibited a clear separation from oligotrophic-dominated to eutrophicdominated assemblages (Figure 3). A higher beta diversity was observed in the OE compared with the other two groups, and the OE also exhibited the lowest alpha diversity (Figures $2 \& 3$ ). This finding may be attributed to the fact that many taxa of Bacillariophyta prefer the relatively low turbidity and low nutrient concentrations in the seawater (Wasmund et al., 2011). As expected, community dissimilarity between different groups exhibited significantly greater variances, indicating greater variances within the communities that exhibited lower alpha diversity. Interestingly, moderate eutrophication caused the selectivity and stability of more convergent SCP communities, thereby resulting in reduced beta diversity

Page $11 / 29$ 
(Figure 3). Additionally, the HE also exhibited lower community dissimilarity, which may be due to aquaculture activities; for example, shellfish farming not only controlled the size of SCP populations but also the growth of certain algal species through their selective filtration (Jiang et al., 2016). Thus, a reduction in the impact of aquaculture activity is an effective method to control the occurrence of microalgae blooms in such a semi-closed and intensively eutrophic gulf. As previous studies reported ( $\mathrm{Li}$ et al., 2016), relatively high temperature stimulated both photosynthesis and respiration, thereby impacting the biodiversity of phytoplankton at the sea surface given its significant effects on the metabolic rate of SCP (Regaudie-De-Gioux and Duarte, 2012). We observed that the variation in SCP community composition was related to a direct influence of temperature, salinity, nitrogen and phosphorus, which displayed stronger significant correlations based on mantel test analysis (Table 1). A previous study also found that SCP community diversity dramatically decreased with the addition of DIN and DIP (Zhou et al., 2018). In other words, high nutrient input could reduce beta diversity driven by phytoplankton competition. In addition, we observed a scale-dependent distance-decay relationship between community similarity and geographic distance in SCP communities (Figure 4), which is similar to observations in bacterial (Martiny et al., 2011) and fungal communities (Zhao et al., 2019). However, our results were in agreement with a previous study (Santos et al., 2016) demonstrating that environmental variables were more important than geographic distance on a regional scale (Figure 4 and Table 1).

\subsection{Co-occurrence networks reveal SCP community dynamics}

We constructed co-occurrence networks to explore interspecies interactions that drove the SCP community responses to eutrophic disturbances that correlated with ecosystem stability (Figure 6). In this study, the inter-domain co-occurrence network exhibited "small-world" features, non-randomly connected properties, and modular structure (Figure $6 \&$ Table S6). The degree and betweenness centrality value described the level of connectedness between ASVs, which provides information about the importance of ASVs to network connectivity. ASVs act as important keystone species in this study that mainly belonged to the OE group, indicating that these taxa may play irreplaceable roles in maintaining SCP community structure (Figure S1). Modularity may reflect synergistic relations, competitive interactions, and niche differentiation, inducing non-random patterns of interaction and the complexity of ecological networks (Olesen et al., 2007). The association of SCP competition and succession played a significant role in altering community structure when ecosystems suffered nutrient variations ( $Y$, 2014). Most modules in our network exhibited distinct eutrophic variation, potentially identifying different groups that performed different functions (Xiong et al., 2017). Specifically, ASVs in module II were specific to the HE. The majority of ASVs in module IV primarily occurred in the ME, while ASVs that were mostly affiliated with Bacillariophyta in modules I and III were specific to the OE (Figure 6b \& S2). Interestingly, the correlations associated with Chlorophyta were greater in these modules (Table S6). Furthermore, network variation was significantly correlated with Euclidean distance of nutrients, which may play an important role in the 
composition and connection of corresponding module. Evidence indicates that increasing eutrophication stress enhances the strength of interactions among species (Thébault and Fontaine, 2010), whereas a densely competitive relationship may exist in the oligotrophic area. These results showed that the complexity of interspecies interaction varied with eutrophic levels because niche conservatism led to the coexistence of closely related SCP taxa. Therefore, the complexity of interspecies interactions was dependent on eutrophic states.

\subsection{Stochastic and deterministic processes shape the SCP community}

It is now generally accepted that deterministic and stochastic processes jointly control the diversity of microbial communities, but uncertainty remains about their relative importance in disturbance intensity (Xiong et al., 2015). In this study, deterministic processes of environmental selection play a more important role than stochastic processes in shaping SCP community assembly at the regional scale of heavy eutrophication (HE), while stochastic processes such as dispersal and drift play a predominant role in structuring SCP communities on large scales (Figure 7). This divergence was attributed to the notion that SCP assemblages are resilient to short-term eutrophication disturbances, whereas continuous disturbances induced environmental selection pressure that become dominant with increasing eutrophic states (Shade et al., 2011). Accordingly, although stochastic processes were therefore dominant in governing SCP community assembly in moderate, oligotrophic areas and the entire Beibu Gulf, the underlying deterministic processes depend on the severity and duration of eutrophic disturbances (Berga et al., 2012). By quantifying the effects of an individual environmental factor, we found that nitrogen concentration (DIN and TDN) was the most important factor associated with environmental selection, especially in the HE (Table 2). Although nutrients exhibited strong selective pressure on the SCP community, it should be noted that NTI decreased with increasing DIN and El, indicating that SCP community assembly was less phylogenetically clustered in increasing eutrophic states (Figure 8). These findings were consistent with many studies demonstrating that environmental filtering is a potentially essential driver of variation in SCP community structure at small spatial scales, whereas dispersal limitation was not strong (Jyrkänkallio-Mikkola et al., 2016). In summary, both deterministic and stochastic processes contribute to SCP community structure and assembly in the Beibu Gulf. Taken together, our study provides valuable insights into how community assembly processes shape biogeographic patterns of SCP communities in the Beibu Gulf.

\section{Conclusion}

SCP community is considered to be one of the indicators of environmental change and ecosystem stability owing to their fast and strong responses to environmental disturbances such as eutrophication in coastal ecosystems. In this study, we found that SCP communities exhibited different and complex compositions, diversity and assembly processes in response to environmental changes under eutrophic states. The genera of Thalassiosira, Pseudo-nitzschia and Skeletonema were significantly over- 
dominated in the HE related to heavy eutrophic state, whereas the ME and OE groups related to medium and oligotrophic states, respectively, were mostly affiliated with Thalassiosira, Minutocellus, Navicula, and Chaetoceros. Both stochastic and deterministic processes simultaneously influenced the assembly of SCP communities along eutrophic states. Specially, stochastic processes dominantly govern governing SCP community assembly, whereas the underlying deterministic processes depend on the severity and duration of eutrophic disturbances. In addition, co-occurrence network analyses revealed that the interspecies interaction of SCP community varied based on different eutrophic states. Our results provide strong evidence for understanding the general pattern of SCP community diversity, assembly and interaction in response to increasing eutrophication.

\section{Declarations}

\section{Acknowledgement}

This study was financially supported by the following funding sources: The National Natural Science Foundation of China (No. 41966005); Guangxi Natural Science Foundation (No. 2018JJD130026); National Research Foundation of Korea (No. 2018R1C1B6007755100); and Plan on the Introduction of High-level Overseas Talents for Colleges and Universities in Guangxi (6020303891251); The Foundation of Guangxi Academy of Sciences under contract 2017YJJ23003.

\section{Declaration of competing interest}

The authors declare that they have no known competing financial interests or personal relationships that could have appeared to influence the work reported in this paper.

\section{References}

Almanza, V., Pedreros, P., Laughinghouse IV, H.D., Félez, J., Parra, O., Azócar, M., Urrutia, R., 2019. Association between trophic state, watershed use, and blooms of cyanobacteria in south-central Chile. Limnologica 75, 30-41. https://doi.org/10.1016/j.limno.2018.11.004

Beaver, J.R., Tausz, C.E., Scotese, K.C., Pollard, A.I., Mitchell, R.M., 2018. Environmental factors influencing the quantitative distribution of microcystin and common potentially toxigenic cyanobacteria in US lakes and reservoirs. Harmful Algae 78, 118-128. https://doi.org/10.1016/j.hal.2018.08.004

Berga, M., Székely, A.J., Langenheder, S., 2012. Effects of disturbance intensity and frequency on bacterial community composition and function. PLoS One 7 (5), e36959.

https://doi.org/10.1371/journal.pone.0036959

Bolaños, L.M., Karp-Boss, L., Choi, C.J., Worden, A.Z., Graff, J.R., Haëntjens, N., Chase, A.P., Della Penna, A., Gaube, P., Morison, F., Menden-Deuer, S., Westberry, T.K., O’Malley, R.T., Boss, E., Behrenfeld, M.J., 
Giovannoni, S.J., 2020. Small phytoplankton dominate western North Atlantic biomass. The ISME Journal 14 (7), 1663-1674. https://doi.org/10.1038/s41396-020-0636-0

Bollobas, B., 1984. The evolution of random graphs. Transactions of the American Mathematical Society 286 (1), 257-274. https://doi.org/10.2307/1999405

Cai, Y., Guo, L., 2009. Abundance and variation of colloidal organic phosphorus in riverine, estuarine, and coastal waters in the northern Gulf of Mexico. Limnol. Oceanogr. 54 (4), 1393-1402.

https://doi.org/10.4319/lo.2009.54.4.1393

Callahan, B.J., McMurdie, P.J., Holmes, S.P., 2017. Exact sequence variants should replace operational taxonomic units in marker-gene data analysis. The ISME Journal 11 (12), 2639-2643.

https://doi.org/10.1038/ismej.2017.119

Capblancq, J., 1990. Nutrient dynamics and pelagic food web interactions in oligotrophic and eutrophic environments: an overview. Hydrobiologia 207 (1), 1-14. https://doi.org/10.1007/BF00041435

Chen, W., Ren, K., Isabwe, A., Chen, H., Liu, M., Yang, J., 2019. Stochastic processes shape microeukaryotic community assembly in a subtropical river across wet and dry seasons. Microbiome 7 (1), 138. https://doi.org/10.1186/s40168-019-0749-8

Csardi, G., Nepusz, T., 2006. The igraph software package for complex network research. InterJournal, complex systems 1695 (5), 1-9.

Dai, W., Zhang, J., Tu, Q., Deng, Y., Qiu, Q., Xiong, J., 2017. Bacterioplankton assembly and interspecies interaction indicating increasing coastal eutrophication. Chemosphere 177, 317-325.

https://doi.org/10.1016/j.chemosphere.2017.03.034

Ding, Y., Xu, H., Deng, J., Qin, B., He, Y., 2019. Impact of nutrient loading on phytoplankton: a mesocosm experiment in the eutrophic Lake Taihu, China. Hydrobiologia 829 (1), 167-187.

https://doi.org/10.1007/s10750-018-3830-6

Dixon, P., 2003. VEGAN, a package of $R$ functions for community ecology. Journal of Vegetation Science 14 (6), 927-930. https://doi.org/10.1111/j.1654-1103.2003.tb02228.x

Escalas, A., Catherine, A., Maloufi, S., Cellamare, M., Hamlaoui, S., Yéprémian, C., Louvard, C., Troussellier, M., Bernard, C., 2019. Drivers and ecological consequences of dominance in periurban phytoplankton communities using networks approaches. Water Res. 163, 114893.

https://doi.org/10.1016/j.watres.2019.114893

Falkowski, P.G., Barber, R.T., Smetacek, V., 1998. Biogeochemical controls and feedbacks on ocean primary production. Science 281 (5374), 200-206. https://doi.org/10.1126/science.281.5374.200 
Ferreira, A., Garrido-Amador, P., Brito, A.C., 2019. Disentangling Environmental Drivers of Phytoplankton Biomass off Western Iberia. Frontiers in Marine Science 6 (44).

https://doi.org/10.3389/fmars.2019.00044

Gao, C., Montoya, L., Xu, L., Madera, M., Taylor, J.W., 2020. Fungal community assembly in droughtstressed sorghum shows stochasticity, selection, and universal ecological dynamics. Nature Communications 11 (1). https://doi.org/10.1038/s41467-019-13913-9

Han, A., Dai, M., Kao, S.-J., Gan, J., Li, Q., Wang, L., Zhai, W., Wang, L., 2012. Nutrient dynamics and biological consumption in a large continental shelf system under the influence of both a river plume and coastal upwelling. Limnol. Oceanogr. 57 (2), 486-502. https://doi.org/10.4319/lo.2012.57.2.0486

Hanson, C.A., Fuhrman, J.A., Horner-Devine, M.C., Martiny, J.B.H., 2012. Beyond biogeographic patterns: processes shaping the microbial landscape. Nature Reviews Microbiology 10 (7), 497-506. https://doi.org/10.1038/nrmicro2795

Harrell Jr, F.E., Dupont, M.C., 2006. The Hmisc Package. R package version 3 (0-12), 3.

Holm-Hansen, O., Riemann, B., 1978. Chlorophyll a determination: improvements in methodology. Oikos438-447.

Hou, F., Zhang, H., Xie, W., Zhou, X., Zhu, X., Zhang, D., 2020. Co-occurrence patterns and assembly processes of microeukaryotic communities in an early-spring diatom bloom. Sci. Total Environ. 711, 134624. https://doi.org/10.1016/j.scitotenv.2019.134624

Irion, S., Jardillier, L., Sassenhagen, I., Christaki, U., 2020. Marked spatiotemporal variations in small phytoplankton structure in contrasted waters of the Southern Ocean (Kerguelen area). Limnol. Oceanogr. 1-18. https://doi.org/10.1002/Ino.11555

Irwin, A.J., Finkel, Z.V., Schofield, O., Falkowski, P.G., 2006. Scaling-up from nutrient physiology to the sizestructure of phytoplankton communities. J. Plankton Res. 28 (5), 459-471.

https://doi.org/10.1093/plankt/fbi148

Jiang, T., Chen, F., Yu, Z., Lu, L., Wang, Z., 2016. Size-dependent depletion and community disturbance of phytoplankton under intensive oyster mariculture based on HPLC pigment analysis in Daya Bay, South China Sea. Environ. Pollut. 219, 804-814. https://doi.org/10.1016/j.envpol.2016.07.058

John, D.E., Patterson, S.S., Paul, J.H., 2007. Phytoplankton-Group Specific Quantitative Polymerase Chain Reaction Assays for RuBisCO mRNA Transcripts in Seawater. Mar. Biotechnol. 9 (6), 747-759. https://doi.org/10.1007/s10126-007-9027-z

Johnson, E., 2019. Gephi-Network analysis and visualization. 
Jyrkänkallio-Mikkola, J., Heino, J., Soininen, J., 2016. Beta diversity of stream diatoms at two hierarchical spatial scales: implications for biomonitoring. Freshwat. Biol. 61 (2), 239-250.

https://doi.org/10.1111/fwb.12697

Kolde, R., 2012. Pheatmap: pretty heatmaps. R package version 61, 617.

Leruste, A., Guilhaumon, F., De Wit, R., Malet, N., Collos, Y., Bec, B., 2019. Phytoplankton strategies to exploit nutrients in coastal lagoons with different eutrophication status during re-oligotrophication. Aquat. Microb. Ecol. 83 (2), 131-146. https://doi.org/10.3354/ame01906

Li, N., Yu, S.-X., Wang, Y.-C., Li, J.-L., Li, F.-C., Qin, S., 2016. Diversity of phototrophic phytoplankton in Northern South China Sea indicated by rbcL analysis. J. Appl. Phycol. 28 (2), 773-781. https://doi.org/10.1007/s10811-015-0624-3

Li, N., Zhao, H., Jiang, G., Xu, Q., Tang, J., Li, X., Wen, J., Liu, H., Tang, C., Kang, Z., 2020. Phylogenetic Responses of marine free-living bacterial Community to Phaeocystis globosa bloom in Beibu Gulf, China. Frontiers in Microbiology 11, 1624. https://doi.org/10.3389/fmicb.2020.01624

Martiny, J.B.H., Eisen, J.A., Penn, K., Allison, S.D., Horner-Devine, M.C., 2011. Drivers of bacterial $\beta$-diversity depend on spatial scale. Proceedings of the National Academy of Sciences 108 (19), 7850-7854. https://doi.org/10.1073/pnas.1016308108

Monchamp, M.-E., Spaak, P., Domaizon, I., Dubois, N., Bouffard, D., Pomati, F., 2018. Homogenization of lake cyanobacterial communities over a century of climate change and eutrophication. Nature ecology \& evolution 2 (2), 317-324. https://doi.org/10.1038/s41559-017-0407-0

Li, N., Yu, S.X., Wang, Y.-C., Li, J.-L., Li, F.-C., 2016. Diversity of phototrophic phytoplankton in Northern South China Sea indicated by rbcL analysis. J. Appl. Phycol. 28, 773-781. 110.1007/s10811-015-0624-3

Olesen, J.M., Bascompte, J., Dupont, Y.L., Jordano, P., 2007. The modularity of pollination networks. Proc Natl Acad Sci U S A 104 (50), 19891-19896. https://doi.org/10.1073/pnas.0706375104

Ribeiro, K.F., da Rocha, C.M., de Castro, D., Rodrigues, L.R., Crossetti, L.O., 2018. Distribution and coexistence patterns of phytoplankton in subtropical shallow lakes and the role of niche-based and spatial processes. Hydrobiologia 814 (1), 233-246. https://doi.org/10.1007/s10750-018-3539-6

Oren, A., 2011. Thermodynamic limits to microbial life at high salt concentrations. Environ. Microbiol. 13 (8), 1908-1923. https://doi.org/10.1111/j.1462-2920.2010.02365.x

Paches, M., Aguado, D., Martínez-Guijarro, R., Romero, l., 2019. Long-term study of seasonal changes in phytoplankton community structure in the western Mediterranean (Valencian Community). Environmental Science and Pollution Research 26 (14), 14266-14276. https://doi.org/10.1007/s11356-019-04660-x

Park, H.M., 2009. Comparing group means: t-tests and one-way ANOVA using Stata, SAS, R, and SPSS. 
Pujari, L., Wu, C., Kan, J., Li, N., Wang, X., Zhang, G., Shang, X., Wang, M., Zhou, C., Sun, J., 2019. Diversity and Spatial Distribution of Chromophytic Phytoplankton in the Bay of Bengal Revealed by RuBisCO Genes (rbcL). Frontiers in Microbiology 10 (1501). https://doi.org/10.3389/fmicb.2019.01501

Regaudie-De-Gioux, A., Duarte, C.M., 2012. Temperature dependence of planktonic metabolism in the ocean. Global Biogeochemical Cycles 26 (1), GB1015. https://doi.org/10.1029/2010GB003907

Revelle, W., Revelle, M.W., 2015. Package 'psych'. The Comprehensive R Archive Network.

Santos, J.B., Silva, L.H., Branco, C.W., Huszar, V.L., 2016. The roles of environmental conditions and geographical distances on the species turnover of the whole phytoplankton and zooplankton communities and their subsets in tropical reservoirs. Hydrobiologia 764 (1), 171-186. https://doi.org/10.1007/s10750-015-2296-z

Shade, A., Read, J.S., Welkie, D.G., Kratz, T.K., Wu, C.H., McMahon, K.D., 2011. Resistance, resilience and recovery: aquatic bacterial dynamics after water column disturbance. Environ. Microbiol. 13 (10), 27522767. https://doi.org/10.1111/j.1462-2920.2011.02546.x

Staay, M.V.D., Staay, G.W.M.V.D., Guillou, L., Vaulot, D., Medlin, C.L.K., 2000. Abundance and Diversity of Prymnesiophytes in the Picoplankton Community from the Equatorial Pacific Ocean Inferred from 18S rDNA Sequences. Limnology \& Oceanography 45 (1), 98-109. https://doi.org/10.4319/lo.2000.45.1.0098

Stegen, J.C., Lin, X., Konopka, A.E., Fredrickson, J.K., 2012. Stochastic and deterministic assembly processes in subsurface microbial communities. Isme Journal 6 (9), 1653-1664.

https://doi.org/10.1038/ismej.2012.22

Thébault, E., Fontaine, C., 2010. Stability of ecological communities and the architecture of mutualistic and trophic networks. Science 329 (5993), 853-856. https://doi.org/10.1126/science.1188321

Tian, W., Zhang, H., Zhao, L., Zhang, F., Huang, H., 2017. Phytoplankton diversity effects on community biomass and stability along nutrient gradients in a Eutrophic Lake. Int. J. Env. Res. Public Health 14 (1), 95. https://doi.org/10.3390/ijerph14010095

Van Der Gast, C.J., Ager, D., Lilley, A.K., 2008. Temporal scaling of bacterial taxa is influenced by both stochastic and deterministic ecological factors. Environ. Microbiol. 10 (6), 1411-1418.

https://doi.org/10.1111/j.1462-2920.2007.01550.x

Visco, G., Campanella, L., Nobili, V., 2005. Organic carbons and TOC in waters: an overview of the international norm for its measurements. Microchemical Journal 79 (1), 185-191.

https://doi.org/10.1016/j.microc.2004.10.018

Wasmund, N., Tuimala, J., Suikkanen, S., Vandepitte, L., Kraberg, A., 2011. Long-term trends in phytoplankton composition in the western and central Baltic Sea. J. Mar. Syst. 87 (2), 145-159. https://doi.org/10.1016/j.jmarsys.2011.03.010

Page $18 / 29$ 
Wentzky, V.C., Tittel, J., Jäger, C.G., Bruggeman, J., Rinke, K., 2020. Seasonal succession of functional traits in phytoplankton communities and their interaction with trophic state. J. Ecol. 108 (4), 1649-1663. https://doi.org/10.1111/1365-2745.13395

Xiong, J., Chen, H., Hu, C., Ye, X., Kong, D., Zhang, D., 2015. Evidence of bacterioplankton community adaptation in response to long-term mariculture disturbance. Scientific Reports 5, 15274. https://doi.org/10.1038/srep15274

Xiong, W., Jousset, A., Guo, S., Karlsson, I., Zhao, Q., Wu, H., Kowalchuk, G.A., Shen, Q., Li, R., Geisen, S., 2017. Soil protist communities form a dynamic hub in the soil microbiome. Isme Journal. 12(2), 634-638. https://doi.org/10. 1038/ismej.2017.171

Xu, H.H., Tabita, F.R., 1996. Ribulose-1,5-bisphosphate carboxylase/oxygenase gene expression and diversity of Lake Erie planktonic microorganisms. Appl. Environ. Microbiol. 62 (6), 1913-1921. https://doi.org/10.1089/oli.1.1996.6.145

Xu, Y., Zhang, T., Zhou, J., 2019. Historical Occurrence of Algal Blooms in the Northern Beibu Gulf of China and Implications for Future Trends. Frontiers in Microbiology 10 (451). https://doi.org/10.3389/fmicb.2019.00451

Xue, Y., Chen, H., Yang, J.R., Liu, M., Huang, B., Yang, J., 2018. Distinct patterns and processes of abundant and rare eukaryotic plankton communities following a reservoir cyanobacterial bloom. The ISME journal 12 (9), 2263-2277.

Y, H., 2014. Research Progress of Competitive Mechanisms Among Microalgae. Environ. Sci. Technol.

Yachi, S., Loreau, M., 1999. Biodiversity and ecosystem productivity in a fluctuating environment: The insurance hypothesis. Proceedings of the National Academy of Sciences of the United States of America 96 (4), 1463-1468. https://doi.org/10.1038/s41396-018-0159-0

Yan, M., Chen, S., Huang, T., Li, B., Li, N., Liu, K., Zong, R., Miao, Y., Huang, X., 2020. Community compositions of phytoplankton and eukaryotes during the mixing periods of a drinking water reservoir: dynamics and interactions. Int. J. Env. Res. Public Health 17 (4), 1128.

https://doi.org/10.3390/ijerph17041128

Zhao, J., Gao, Q., Zhou, J., Wang, M., Liang, Y., Sun, B., Chu, H., Yang, Y., 2019. The scale dependence of fungal community distribution in paddy soil driven by stochastic and deterministic processes. Fungal Ecology 42, 100856. https://doi.org/10.1016/j.funeco.2019.07.010

Zhou, J., Deng, Y., Zhang, P., Xue, K., Liang, Y., Van Nostrand, J.D., Yang, Y., He, Z., Wu, L., Stahl, D.A., 2014. Stochasticity, succession, and environmental perturbations in a fluidic ecosystem. Proceedings of the National Academy of Sciences 111 (9), 836-845. https://doi.org/10.1073/pnas.1324044111 
Zhou, Y., Hu, B., Zhao, W., Cui, D., Tan, L., Wang, J., 2018. Effects of increasing nutrient disturbances on phytoplankton community structure and biodiversity in two tropical seas. Mar. Pollut. Bull. 135, 239-248. https://doi.org/10.1016/j.marpolbul.2018.07.033

Zhou, Y., Zhang, Y., Li, F., Tan, L., Wang, J., 2017. Nutrients structure changes impact the competition and succession between diatom and dinoflagellate in the East China Sea. Sci. Total Environ. 574, 499-508. https://doi.org/10.1016/j.scitotenv.2016.09.092

\section{Tables}

Table 1. The mantel test (Pearson correlations) showed the correlations between $\beta$-diversity of SCP communities (Bray-Curtis dissimilarity) and environmental factors (Euclidean distance) with 999 permutations. $(*: p<0.05, * *: p<0.01, * * *: p<0.001)$

\begin{tabular}{|c|c|c|c|c|}
\hline Variables & All & High & Medium & Oligo \\
\hline Geo-distance & $0.488^{* * *}$ & $0.629 * * *$ & $0.413^{* * *}$ & $0.397 * * *$ \\
\hline Env-factors & $0.603^{* * *}$ & $0.622 * * *$ & $0.629 * * *$ & $0.475^{* * *}$ \\
\hline Temp & $0.447 * * *$ & 0.133* & $0.149 * *$ & 0.038 \\
\hline $\mathrm{pH}$ & $0.435^{* * *}$ & $0.187 *$ & $0.369 * * *$ & $0.170 * *$ \\
\hline Salinity & $0.403^{* * *}$ & $0.619 * * *$ & $0.324 * * *$ & $0.359 * * *$ \\
\hline $\mathrm{NO}_{2}^{-}$ & $0.509 * * *$ & $0.770 * * *$ & $0.260 * * *$ & $0.542^{* * *}$ \\
\hline $\mathrm{NO}_{3}^{-}$ & $0.437 * * *$ & $0.690 * * *$ & $0.450 * * *$ & $0.288^{* * *}$ \\
\hline $\mathrm{NH}_{4}^{+}$ & $0.324^{* * *}$ & $0.552^{* * *}$ & $0.400^{* * *}$ & $0.608^{* * *}$ \\
\hline Chl a & $0.261 * * *$ & $0.581^{* * *}$ & $0.302 * * *$ & $0.400 * * *$ \\
\hline DIN & $0.370^{* * *}$ & $0.568 * * *$ & $0.363^{* * *}$ & $0.269 * * *$ \\
\hline TDN & $0.288 * * *$ & $0.391 * * *$ & $0.250 * * *$ & 0.030 \\
\hline DIP & $0.520^{* * *}$ & $0.759 * * *$ & $0.417 * * *$ & $0.304 * * *$ \\
\hline TDP & $0.520^{* * *}$ & $0.760 * * *$ & $0.431 * * *$ & $0.390 * * *$ \\
\hline TOC & $0.132^{* * *}$ & $0.458^{* * *}$ & $0.372^{* * *}$ & $0.258 * * *$ \\
\hline COD & $0.166^{* * *}$ & $0.548^{* * *}$ & $0.450 * * *$ & $0.199 * *$ \\
\hline EI & $0.371 * * *$ & $0.368 * *$ & $0.552^{* * *}$ & $0.492^{* * *}$ \\
\hline
\end{tabular}


Table 2. The mantel test (Pearson correlations) showed the correlations between $\beta$ NTI and environmental factors (Euclidean distance) with 999 permutations. (*: $p<0.05, * *: p<0.01, * * *: p<0.001$ )

\begin{tabular}{|c|c|c|c|c|}
\hline Variables & All & High & Medium & Oligo \\
\hline Geo-distance & 0.041 & $0.594 * * *$ & 0.079 & -0.070 \\
\hline Env-factors & $0.516^{* * *}$ & $0.952 * * *$ & $0.332 * * *$ & $-0.001 * * *$ \\
\hline Temp & $0.300 * * *$ & -0.016 & -0.128 & 0.098 \\
\hline $\mathrm{pH}$ & -0.221 & $0.658 * * *$ & $0.570 * * *$ & 0.044 \\
\hline Salinity & $0.520 * * *$ & $0.378 * *$ & $0.530 * * *$ & 0.016 \\
\hline $\mathrm{NO}_{2}^{-}$ & 0.068* & 0.166 & $0.544^{* * *}$ & 0.043 \\
\hline $\mathrm{NO}_{3}^{-}$ & $0.635^{* * *}$ & $0.249 *$ & $0.135^{*}$ & -0.095 \\
\hline $\mathrm{NH}_{4}{ }^{+}$ & $0.312^{* * *}$ & $0.867 * * *$ & $0.323^{* * *}$ & 0.016 \\
\hline Chl a & 0.053 & $0.656^{* * *}$ & $0.426^{* * *}$ & -0.011 \\
\hline DIN & $0.665^{* * *}$ & $0.721 * * *$ & $0.490^{* * *}$ & -0.112 \\
\hline TDN & -0.008 & $0.971 * * *$ & $0.530 * * *$ & $0.244 * * *$ \\
\hline DIP & -0.164 & 0.126 & $0.475^{* * *}$ & 0.057 \\
\hline TDP & -0.172 & 0.083 & $0.396^{* * *}$ & 0.056 \\
\hline TOC & 0.030 & $0.361 * *$ & $0.314^{* * *}$ & 0.016 \\
\hline COD & 0.028 & $0.556^{* * *}$ & $0.471^{* * *}$ & -0.082 \\
\hline EI & $0.469 * * *$ & $0.966^{* * *}$ & -0.172 & $0.160 * *$ \\
\hline
\end{tabular}

Figures 


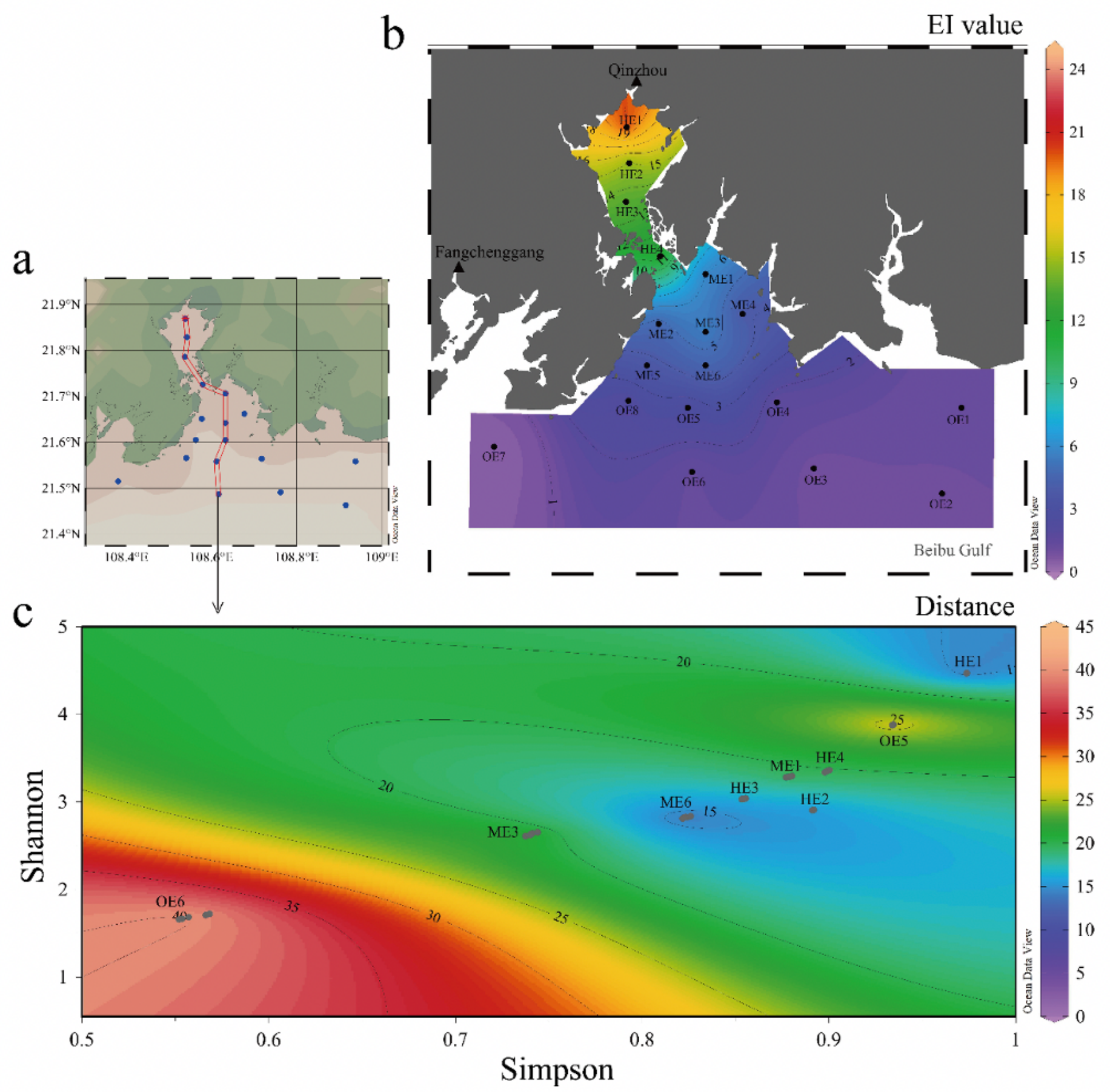

Figure 1

Map of the Beibu Gulf showing the sampling sites using the Ocean Data View. a: A total of 18 sampling sites with five duplicates per site were collected from the Maowei Sea, Qinzhou Bay and Beibu Gulf open sea in November 2017. b: The sampling sites were divided into three groups (HE, ME, and OE) according the El values, which were displayed using different colors on contours map. c: The section of the contours map of nine sites along the distance from estuary to open sea that reflected the variation of Shannon and Simpson indices along the distance. 

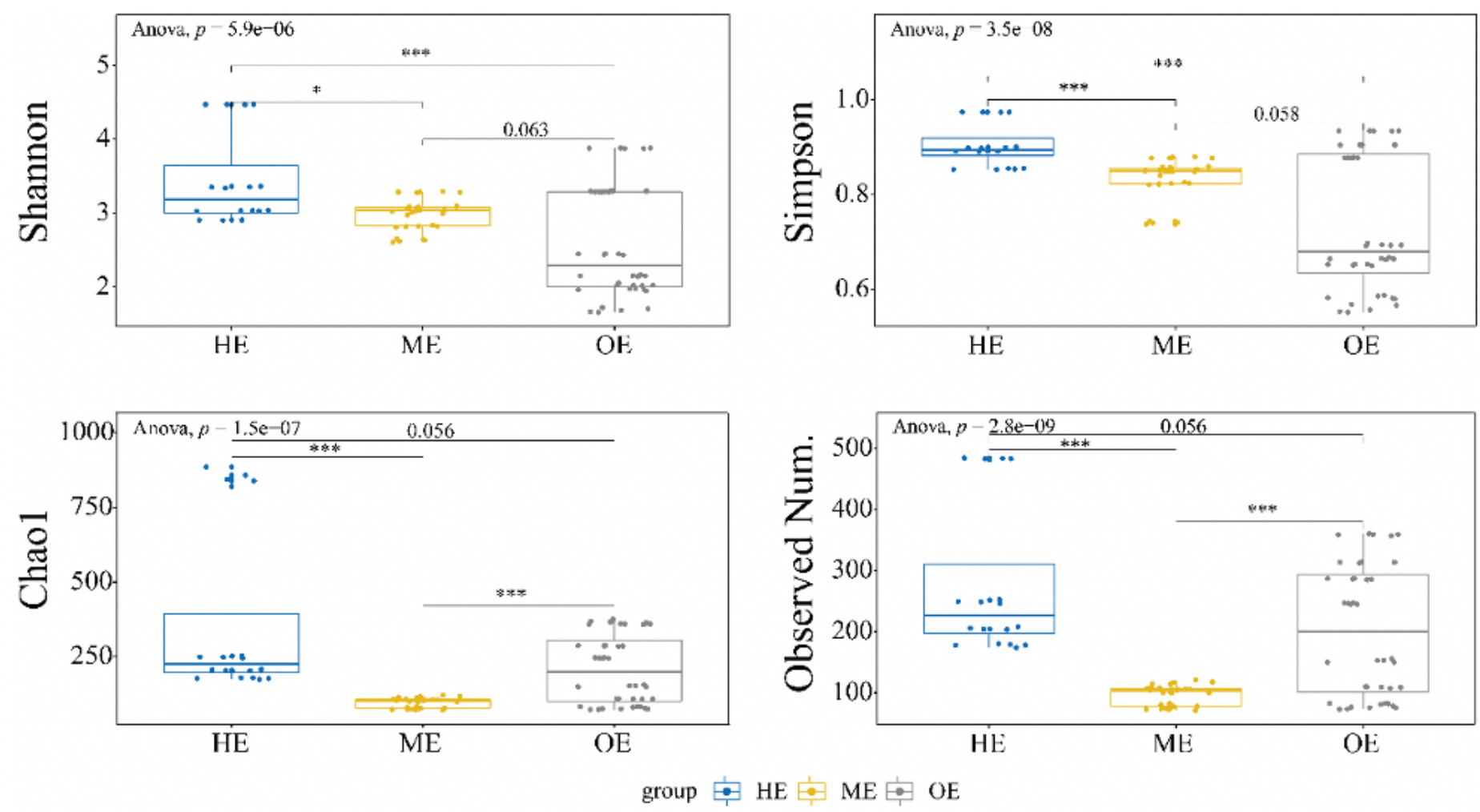

Figure 2

The a-diversity indices (Shannon, Simpson, Chao 1, and Observed Number of ASVs) along eutrophic gradients presented by boxplot. The significant difference in a-diversity indices between groups was calculated using one-way ANOVA. $(*: p<0.05, * \star: p<0.01, \star \star \star: p<0.001)$
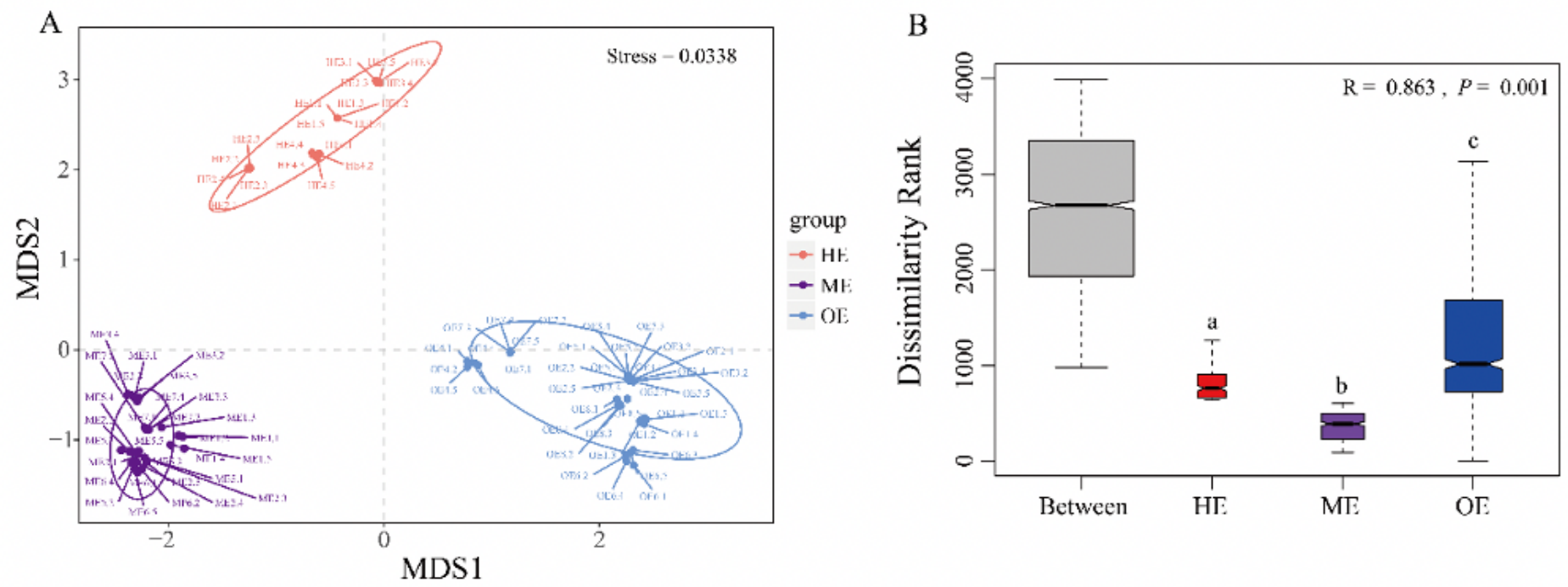

Figure 3 
(a) The $\beta$-diversity was calculated based on Bray-Curtis dissimilarity index and visualized by nonmetric multidimensional scaling (NMDS) and (b) the analysis of similarities (ANOSIM), which was performed to analysis similarity variation in the SCP community.
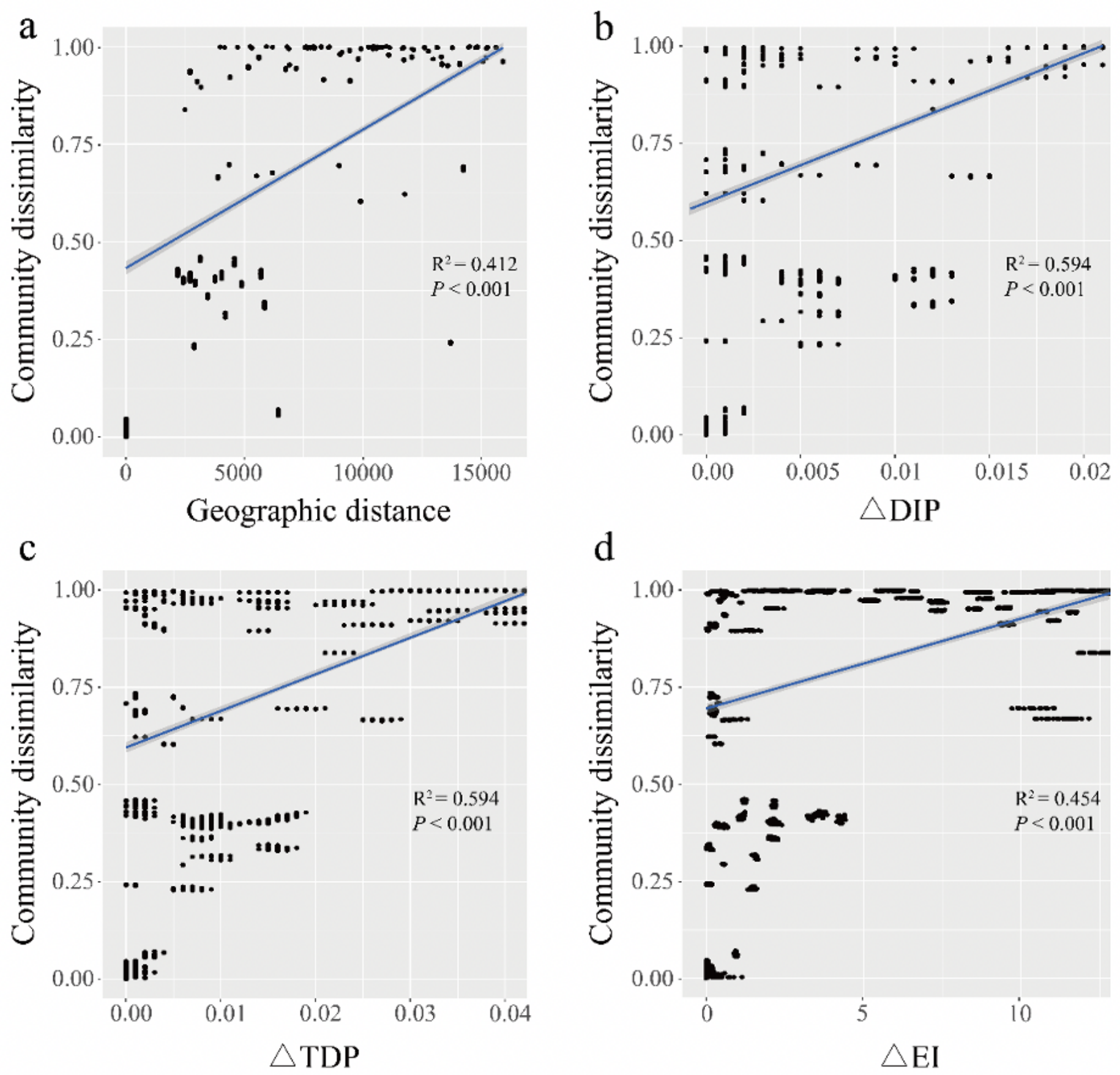

d

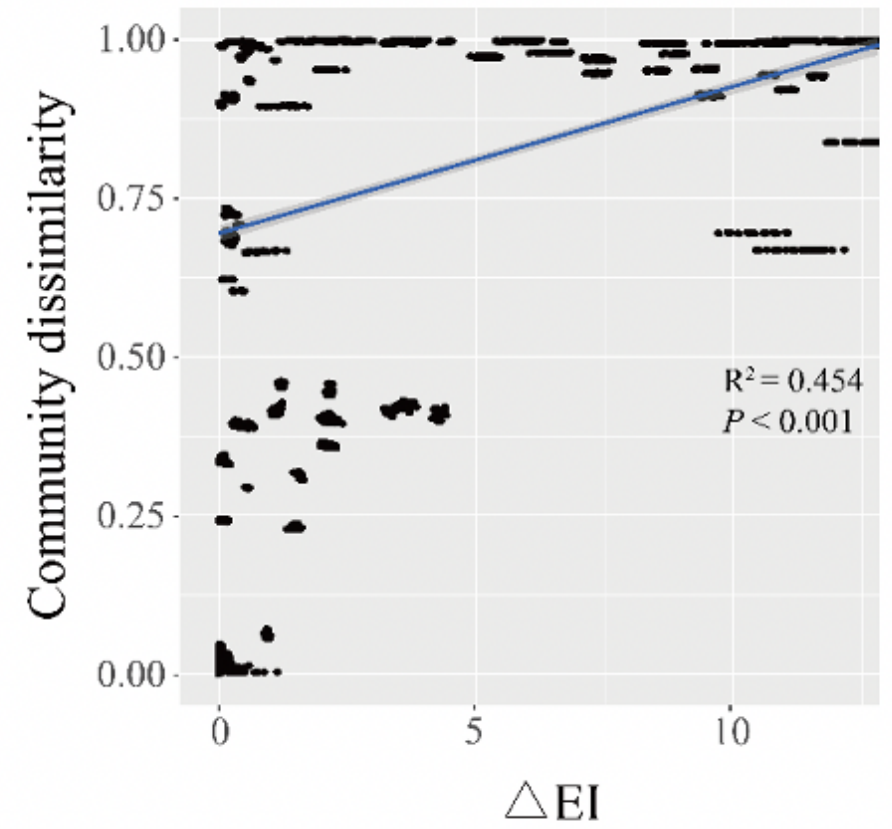

Figure 4

Relationship between Bray-Curtis dissimilarity and differences in geographical distance (a), DIP (b), TDP (c), and El (d), which were calculated based on Euclidean distance. The blue line represents the linear regression, and $p$-values were calculated to indicate significant differences. 


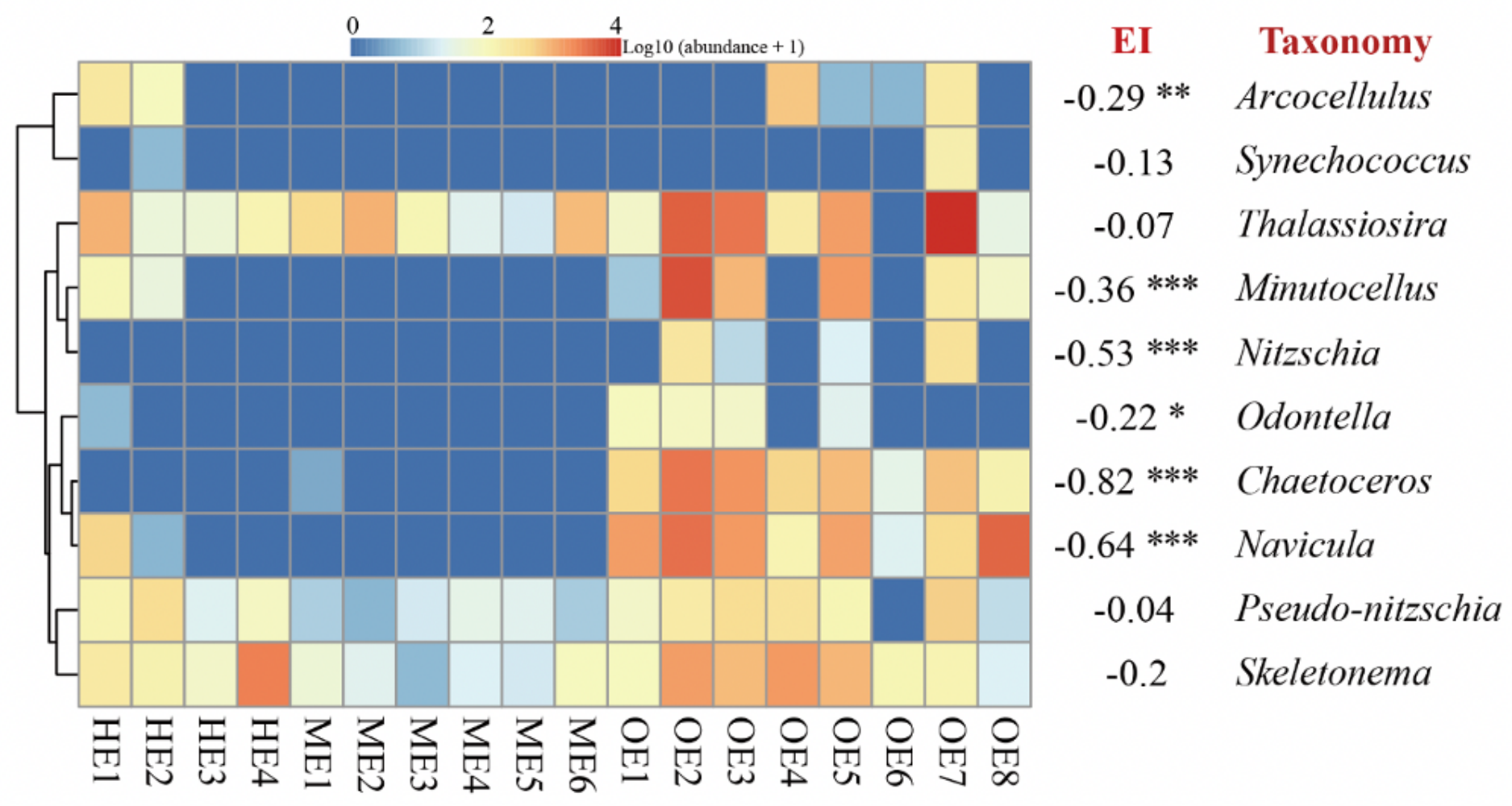

Figure 5

Heat map showing the relative abundance of top $10 \mathrm{SCP}$ community genera along eutrophic gradients and Spearman's rank correlation of these genera with El values (Five duplicates per site). (*: $p<0.05, * \star$ : $p$ $<0.01, * \star *: p<0.001)$ 


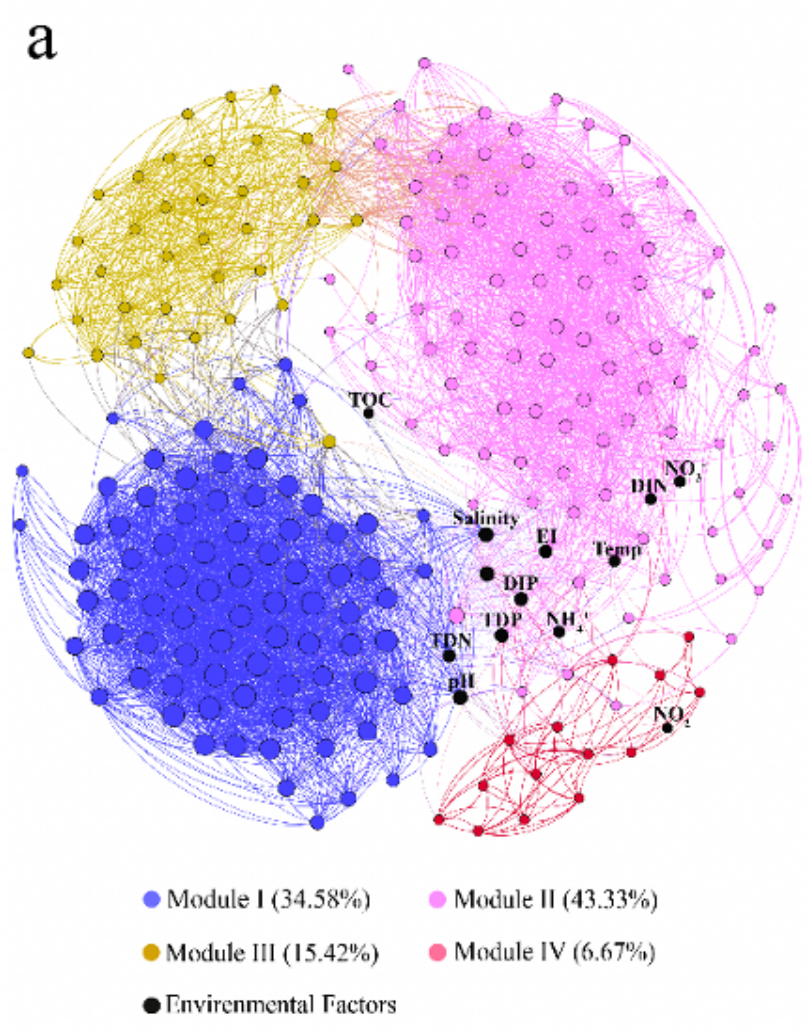

b

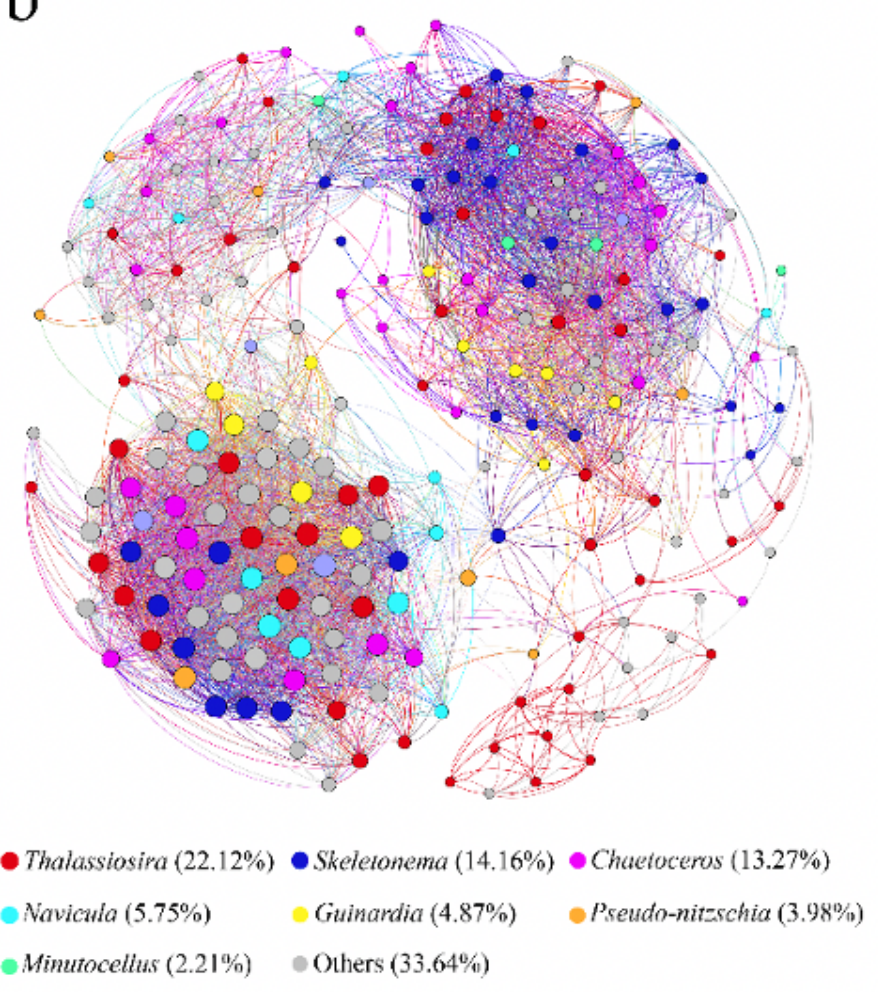

Figure 6

Co-occurrence patterns of SCP communities at the genus level. Node colors indicate the different modularity classes (a) and different taxa (b). A connection represents a strong (Spearman's $|p|>0.60$ ) and significant (FDR-adjusted $\mathrm{P}<0.05$ ) correlation between SCP community in modules and environmental factors. The size of each node is proportional to the eigenvector centrality. 


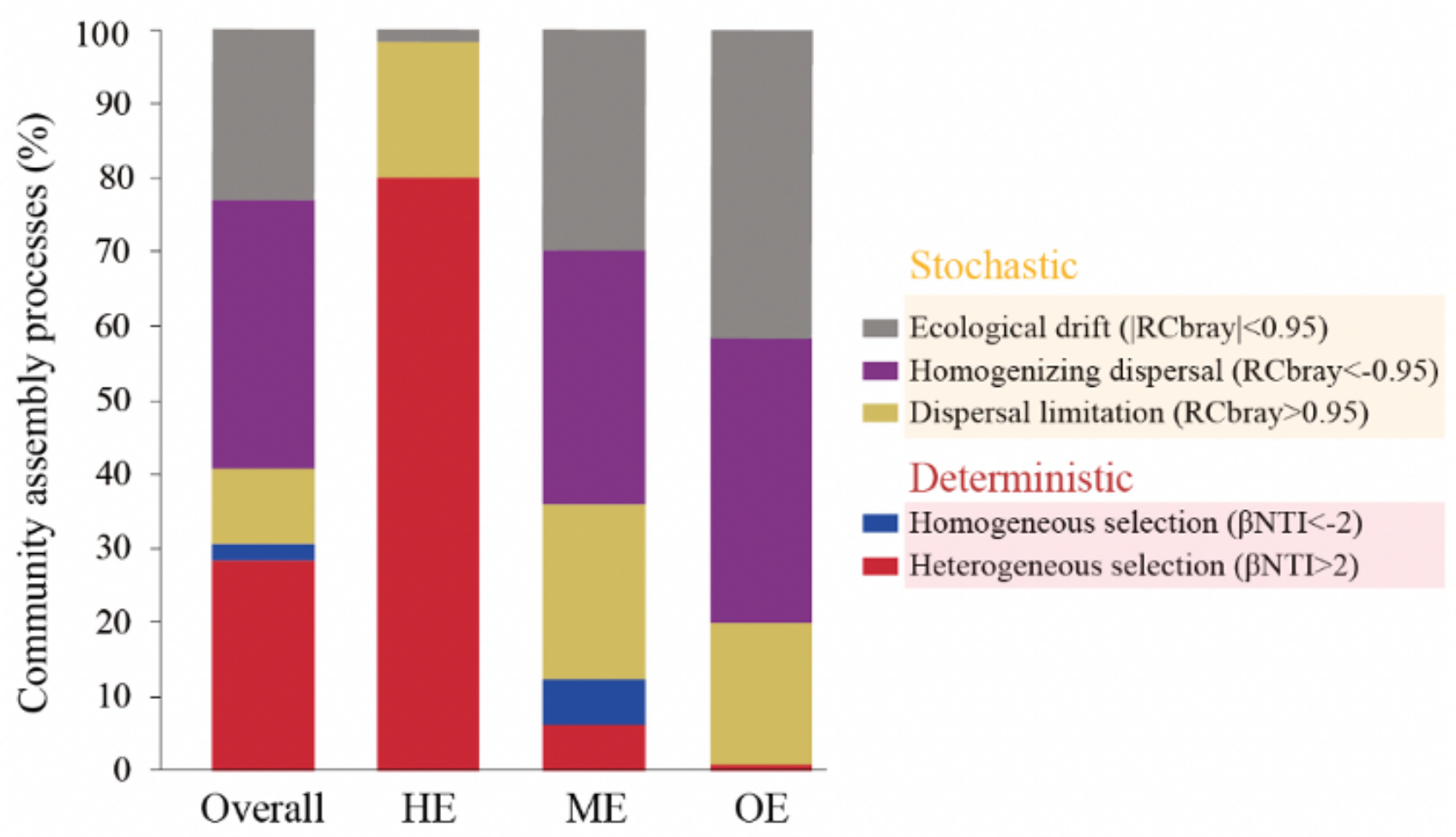

Figure 7

The percent of turnover in SCP community assembly governed primarily by various deterministic, including homogeneous and variable selection, and stochastic processes, including dispersal limitations, homogenizing dispersal, and ecology draft, in all groups and three groups. 

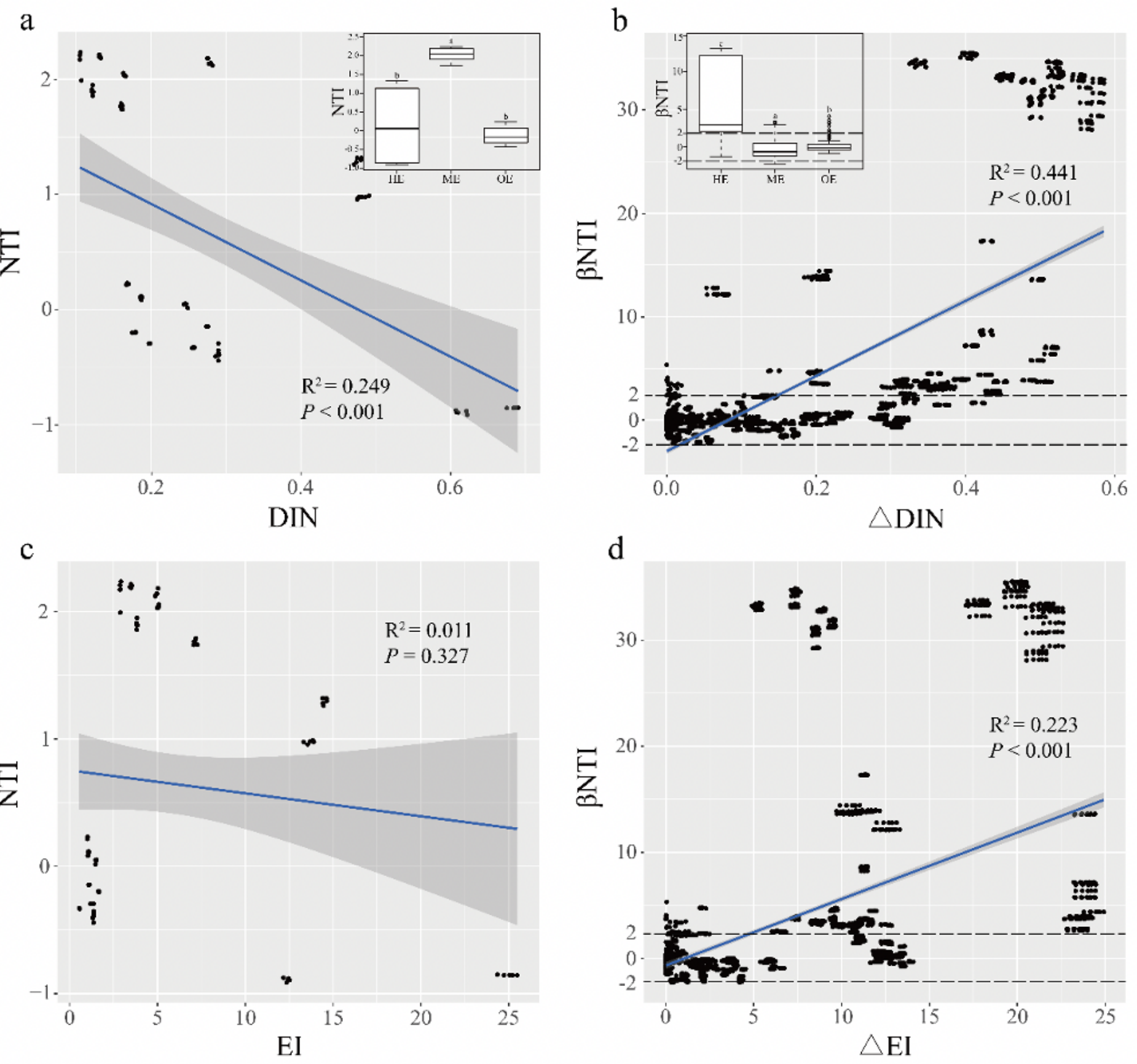

\section{Figure 8}

The relationship among NTI, DIN, and EI for SCP community (a). The relationship between $\beta N T I$ and change in DIN and El which was calculated by Euclidean distance (b). Linear regressions models and associated correlation coefficients are provided on each panel. Horizontal dashed black lines indicate upper and lower significance thresholds at $\beta \mathrm{NTI}=+2$ and -2 , respectively. Boxplots within the panels showed the variation in NTI and $\beta N T I$ in three groups. Boxplots that do not share a letter are significantly different $(p<0.05$; multiple comparisons with Kruskal-Wallis).

\section{Supplementary Files}


This is a list of supplementary files associated with this preprint. Click to download.

- SupplementaryTables.docx

- SupplementalFigures.docx 